\title{
The ab initio model potential method with the spin-free relativistic scheme by eliminating small components Hamiltonian
}

\author{
Kyosuke Motegi, Takahito Nakajima, and Kimihiko Hirao ${ }^{\text {a) }}$ \\ Department of Applied Chemistry, Graduate School of Engineering, The University of Tokyo, \\ Tokyo 113-8656, Japan \\ Luis Seijo \\ Departamento de Química and Instituto Universitario de Ciencia de Materiales Nicolás Cabrera, C-XIV, \\ Universidad Autónoma de Madrid, E-28049 Madrid, Spain
}

(Received 7 December 2000; accepted 30 January 2001)

\begin{abstract}
A relativistic ab initio model potential (AIMP) for $\mathrm{Pt}, \mathrm{Au}$, and $\mathrm{Hg}$ atoms has been developed using a relativistic scheme by eliminating small components (RESC) in which the $5 p, 5 d$, and $6 s$ electrons are treated explicitly. The quality of new RESC-AIMP has been tested by calculating the spectroscopic properties of the hydrides of these elements using the Hartree-Fock and coupled cluster with singles and doubles (CCSD) methods. The agreement with reference all-electron RESC calculations is excellent. The RESC-AIMP method is applied successfully in the investigation of the spectroscopic constants of $\mathrm{Au}_{2}$ and $\mathrm{Hg}_{2}$ using the CCSD method with a perturbative estimate of the contributions of triples. The ground state of $\mathrm{Pt}_{2}$ is also determined by RESC-AIMP with the second-order complete active space perturbation method. The results show that scalar relativistic effects on the valence properties are well described by the RESC-AIMP method. The effect on the basis set superposition error on the spectroscopic constants is also examined. (C) 2001 American
\end{abstract} Institute of Physics. [DOI: 10.1063/1.1356735]

\section{INTRODUCTION}

It is common knowledge that relativistic effects are important in the study of systems that include the heavier elements. The most widely used quantum chemical method in heavy element chemistry is the effective core potential (ECP) approximation. In this approach, the core electrons are modeled using a suitable function, and only the valence electrons are treated explicitly. Part of the relativistic effects, especially the scalar effects, may also be taken into account without having to perform full relativistic calculations. In many cases, this provides quite good results at a reduced cost compared to an all-electron calculation. There are two ECP families: the pseudopotential (PP) method and the model potential (MP) method. In the PP method, derived from the Phillips-Kleinman equation, ${ }^{1}$ the valence orbitals are replaced by a set of nodeless pseudo-orbitals. The valence orbitals have a series of radial nodes in order to make them orthogonal to the core orbitals, and the pseudo-orbitals are designed so that they behave correctly in the outer region, but do not have a nodal structure in the core region. On the other hand, the MP method, originally proposed by Huzinaga and co-workers, ${ }^{2}$ which finally gave rise to the $a b$ initio model potential method (AIMP), ${ }^{3,4}$ describes the correct behavior for the inner nodal structure of the valence orbitals. Since the relativistic operators act in the near vicinity of the nucleus, the MP method might be preferable. However, the MP method requires more primitive basis functions than the PP method in order to represent the correct nodal properties of the valence orbitals.

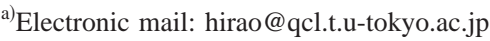

The AIMP consists of a Coulomb potential, an exchange potential, and a projection operator, and has a clear physical meaning, in that it represents Coulombic and exchange interactions between a single valence electron and the core electrons. Several relativistic variations of the AIMP method have been proposed: the AIMP method with the CowanGriffin Hamiltonian (CG-AIMP) ${ }^{5-7}$ the AIMP with the Wood-Boring Hamiltonian-(WB-AIMP), ${ }^{8,9}$ and the AIMP with the Douglas-Kroll transformed no-pair Hamiltonian (NP-AIMP). ${ }^{10-12}$ Remarkable success has been achieved with these relativistic methods for systems containing heavy atoms.

Recently we have proposed a new relativistic scheme by eliminating the small components (RESC) ${ }^{13-17}$ of the fourcomponent Dirac equation. This is variationally stable and avoids the Coulomb singularity. The formalism can easily be applied to any electronic structure theory. The implementation of the spin-free RESC Hamiltonian into nonrelativistic $a b$ initio and density functional theory programs requires only a modification of the one-electron integrals. The RESC can be extended to include the electron correlation by methods analogous to those for the nonrelativistic case. The RESC approach has been proven to yield a satisfying result for valence properties.

In this paper, we propose using the AIMP method with the RESC Hamiltonian (RESC-AIMP) by replacing the nonrelativistic one-electron operator with the spin-free RESC one-electron operator, and have applied this to $\mathrm{Pt}, \mathrm{Au}$, and $\mathrm{Hg}$ atoms. Illustrative calculations are performed for the atomic properties and spectroscopic constants of the hydrides of these elements using the Hartree-Fock (HF) and coupled 
cluster with singles and doubles (CCSD) methods. The differences between the RESC-AIMP and the all-electron RESC are examined to check the quality of the AIMP. The spectroscopic constants for $\mathrm{Au}_{2}, \mathrm{Hg}_{2}$, and $\mathrm{Pt}_{2}$ are also calculated using a large basis set and the highly correlated methods such as CCSD method perturbatively corrected for triples $(\operatorname{CCSD}(\mathrm{T}))$ and the second-order complete active space perturbation (CASPT2) methods.

In Sec. II we briefly describe the theoretical background. Computational details are given in Sec. III. In Sec. IV we present and discuss our calculated results. A conclusion is given in the Sec. V.

\section{THEORETICAL BACKGROUND}

\section{A. The RESC method}

The RESC Hamiltonian can be separated into a spin-free (sf) component and a spin-dependent (sd) component as

$$
H_{\mathrm{RESC}}=H_{\mathrm{RESC}}^{\mathrm{sf}}+H_{\mathrm{RESC}}^{\mathrm{sd}},
$$

where

$$
\begin{aligned}
H_{\mathrm{RESC}}^{\mathrm{sf}}= & \sum_{j} T_{j}+O_{j} Q_{j} \mathbf{p}_{j} \cdot V(j) \mathbf{p}_{j} Q_{j} O_{j}^{-1} \\
& +2 m c O_{j} Q_{j}^{1 / 2} V(j) Q_{j}^{1 / 2} O_{j}^{-1}
\end{aligned}
$$

and

$$
H_{\mathrm{RESC}}^{\mathrm{sd}}=i \sum_{j} O_{j} Q_{j} \sigma \cdot\left(\mathbf{p}_{j} V(j)\right) \times \mathbf{p}_{j} Q_{j} O_{j}^{-1} .
$$

Here, the $O_{j}, Q_{j}$, and $T_{j}$ operators are defined by

$$
\begin{aligned}
& O_{j}=\frac{1}{E_{j}+m c^{2}}\left[1+\frac{p_{j}^{2} c^{2}}{\left(E_{j}+m c^{2}\right)^{2}}\right]^{1 / 2}, \\
& Q_{j}=\frac{1}{E_{j}+m c^{2}},
\end{aligned}
$$

and

$$
T_{j}=\sqrt{m^{2} c^{4}+p_{j}^{2} c^{2}}-m c^{2}
$$

with

$$
E_{j}=\sqrt{m^{2} c^{4}+p_{j}^{2} c^{2}} .
$$

The parameter $\sigma$ denotes the three Pauli $2 \times 2$ spin matrices, and $\mathbf{p}_{i}$ is the momentum operator. For a practical calculation, the Hamiltonian matrix elements are evaluated in the space spanned by the eigenfunctions of the square of the momentum $p^{2}$, following Buenker et al. ${ }^{18}$ The symmetry of $H_{\text {RESC }}$ is Hermitian for mathematical convenience, rather than for physical significance.

The RESC method is variationally stable, and can be easily implemented in various nonrelativistic ab initio theories. Thus, one can handle the relativistic effect on the same footing as electron correlation effects. The detailed derivation and the practical computational techniques are given in Ref. 13.

\section{B. The RESC-AIMP method}

The atomic RESC-AIMP Hamiltonian for $n$ valence electrons is written as

$$
H^{\mathrm{RESC}-\mathrm{AIMP}}=\sum_{i=1}^{n} h(i)+\sum_{i<j}^{n} \frac{1}{r_{i j}},
$$

where

$$
\begin{aligned}
h(i)= & T_{i}+O_{i} Q_{i} \mathbf{p}_{i} \cdot V(i) \mathbf{p}_{i} Q_{i} O_{i}^{-1} \\
& +2 m c O_{i} Q_{i}^{1 / 2} V(i) Q_{i}^{1 / 2} O_{i}^{-1}+\frac{Z_{\text {core }}}{r_{i}} \\
& +V_{\text {Coul }}(i)+V_{\text {exch }}(i)+P(i) .
\end{aligned}
$$

The first and second terms in the one-electron operator are taken from the $H_{\mathrm{RESC}}^{\mathrm{sf}}$ operator. The $V_{\mathrm{Coul}}$ operator represents the Coulomb interactions of one valence electron with the $Z_{\text {core }}$ core electrons and the same number of protons located at the nucleus. It is convenient to fit $V_{\text {Coul }}$ with a linear combination of Gaussian functions

$$
\begin{aligned}
V_{\text {Coul }}(i) & =-\frac{Z_{\text {core }}}{r_{i}}+2 \sum_{c}^{\text {core }} J_{c}(i) \\
& \approx V_{\text {Coul }}^{\mathrm{MP}}(i)=\sum_{j} C_{j} \frac{\exp \left(-\alpha_{j} r_{i}^{2}\right)}{r_{i}} .
\end{aligned}
$$

In the same way, the $V_{\text {exch }}$ operator represents the exchange interaction between one valence electron and the core electrons. This operator is replaced by its spectral representation operator

$$
\begin{aligned}
V_{\mathrm{exch}}(i) & =-\sum_{c}^{\text {core }} K_{c}(i) \\
& \approx V_{\mathrm{exch}}^{\mathrm{MP}}(i)=\sum_{l} \sum_{m=-l}^{l} \sum_{a, b}|a ; \operatorname{lm}\rangle A_{l ; a b}\langle b ; l m|,
\end{aligned}
$$

where $a$ and $b$ are arbitrary Gaussian functions. $A_{l, a b}$ is an element in the following matrix:

$$
A=S^{-1} K S^{-1} \text {, }
$$

where

$$
K_{i j}=\left\langle i\left|V_{\operatorname{exch}}\right| j\right\rangle,
$$

and

$$
S_{i j}=\langle i \mid j\rangle .
$$

By using these operators, all the one-electron exchange integrals become those of the all-electron calculation, if $\langle i|$ and $|j\rangle$ are set to belong to the $\{a ; \operatorname{lm}\}$ and $\{b ; \operatorname{lm}\}$ basis functions.

The operator $P(i)$ is obtained by the core-valence orthogonality, and consists of the core orbitals $\phi_{c}$ and the core orbital energies $\epsilon_{c}$. The nodal structures of the valence orbitals are realized through this operator.

$$
P(i)=-\sum_{c}^{\text {core }} 2 \epsilon_{c}\left|\phi_{c}\right\rangle\left\langle\phi_{c}\right| .
$$


The uncontracted $(22 s 18 p 14 d 9 f)$ basis sets by Fægri ${ }^{11,19}$ were employed as the reference all-electron basis sets. The HF calculations with the all-electron RESC method for $\mathrm{Pt}, \mathrm{Au}$, and $\mathrm{Hg}$ atoms were performed with these basis sets. The core orbitals, which were obtained from those calculations, were used to construct the $V_{\text {Coul }}(i), V_{\text {exch }}(i)$, and $P(i)$ operators. The $5 p, 5 d$, and $6 s$ electrons were treated explicitly. In the fitting process, the parameters $C$ and $\alpha$ in Eqs. (10) were determined using a least-square fit method. As the basis of the spectral representation operator, we employed the whole set of atomic valence primitive functions in all cases.

\section{COMPUTATIONAL DETAILS}

In the atomic calculations, the Fægri $(22 s 18 p 14 d 9 f)$ basis sets ${ }^{11,19}$ were employed in an uncontracted manner. The calculations were performed at the levels of HF and CCSD. The $5 p, 5 d$, and $6 s$ electrons were correlated in the CCSD calculations.

In the calculations of the hydride molecules, all-electron calculations were performed using the Fægri $(22 s 18 p 14 d 9 f)$ basis set. Since the spectroscopic constants are rather sensitive to the outer region of a basis set, the outermost three $s$ and $d$, four $p$, and one $f$ primitive functions were replaced by the corresponding three $s, p, d$ and one $f$ functions of the CG-AIMP $(13 s 10 p 9 d 6 f)$ basis set; ${ }^{7}$ it must be noted that the primitive functions of the CG-AIMP valence basis set have been proven to be useful in NP-AIMP calculations. ${ }^{11,12}$ The AIMP calculations were carried out using the uncontracted basis sets $(22 s 17 p 14 d 9 f)$ and $(13 s 10 p 9 d 6 f)$. The general contracted basis set was also used for the AIMP calculations. The exponents of the valence basis sets used in AIMP calculations are usually fewer than in the respective all-electron calculations, and they are variationally optimized by minimization of the AIMP valence energy. In the present work, however, the entire allelectron primitive expansion was used for the description of the valence electrons since we are now focusing our attention only on the core model potentials. The $(22 s 17 p 14 d 9 f)$ primitive basis set was contracted to a $[4 s 4 p 4 d 2 f]$ set, using self-consistent field coefficients for $s, p$, and $d$ functions. The contraction coefficients of the $f$ function are obtained from the natural orbitals of modified coupled-pair functional $(\mathrm{MCPF})^{20}$ calculations of an atom. The outermost three $s, p$, $d$, and one $f$ functions were left uncontracted. The $(9 s 2 p) /[6 s 2 p]$ set of Lie-Clementi ${ }^{21}$ was used for the $\mathrm{H}$ atom. In the CCSD calculations, $5 p, 5 d$, and $6 s$ electrons of the metal and the $1 s$ electron of the hydrogen were correlated. The correction of the basis set superposition errors (BSSEs) was made using the counterpoise method of Boys and Bernardi. ${ }^{22}$

In the calculations of $\mathrm{Au}_{2}$, the basis set (21s 17p 11d $f f$ ) by Pizlo et al. ${ }^{23}$ was employed. For $\mathrm{Pt}_{2}$ and $\mathrm{Hg}_{2}$, the original basis sets by Fægri were modified. The innermost one $s, p, d$, and two $f$ functions were removed and the outer $14 s 14 p 11 d$ and $15 s 14 p 11 d$ functions were replaced by even-tempered $16 s 14 p 11 d$ and $18 s 14 p 12 d$ functions for $\mathrm{Pt}$ and $\mathrm{Hg}$, respectively. Furthermore, one $f$ function was augmented for each set. Finally, $(23 s 17 p 13 d 8 f)$ and $(24 s 17 p 14 d 8 f)$ basis sets
TABLE I. Radial expectation values in a.u.

\begin{tabular}{lccc}
\hline \hline \multicolumn{1}{c}{ Method } & $5 p$ & $5 d$ & $6 s$ \\
\hline Pt & & & \\
$\quad$ RESC-AE & 1.115 & 1.642 & 3.119 \\
RESC-AIMP & 1.115 & 1.643 & 3.120 \\
$\quad$ NR-AIMP & 1.164 & 1.608 & 3.646 \\
Au & & & \\
$\quad$ RESC-AE & 1.082 & 1.583 & 3.089 \\
RESC-AIMP & 1.082 & 1.583 & 3.090 \\
$\quad$ NR-AIMP & 1.133 & 1.544 & 3.639 \\
Hg & & & \\
RESC-AE & 1.047 & 1.469 & 2.865 \\
RESC-AIMP & 1.047 & 1.469 & 2.866 \\
NR-AIMP & 1.095 & 1.433 & 3.287 \\
\hline \hline
\end{tabular}

${ }^{a}$ NR-AIMP; Nonrelativistic ab initio model potential method.

were obtained for the $\mathrm{Pt}$ and $\mathrm{Hg}$ atoms, respectively. Valence-only atomic natural orbitals (ANO) ${ }^{24-26}$ basis sets were constructed. That is, the natural orbital coefficients from the averaged density matrices of some low-lying states of an atom, calculated by MCPF, were used to derive the contracted valence-only ANO basis sets. For the Au atom, $\mathrm{Au}\left(s^{1} d^{10}\right), \mathrm{Au}\left(s^{2} d^{9}\right)$, and $\mathrm{Au}^{+}\left(s^{0} d^{10}\right)$ states were averaged with weightings 2,1 , and 1 , respectively. Similarly, the $\mathrm{Hg}\left(s^{2} d^{10}\right)$ and $\mathrm{Hg}^{+}\left(s^{1} d^{10}\right)$ states were averaged with weightings 2 and 1 , respectively, for the $\mathrm{Hg}$ basis sets, and three $\operatorname{Pt}\left(s^{1} d^{9}\right), \operatorname{Pt}\left(s^{2} d^{8}\right)$, and $\mathrm{Pt}^{+}\left(s^{0} d^{9}\right)$ states with weightings 2,1 , and 1 , respectively, were averaged for a Pt atom.

All-electron calculations were performed using the MOLPRO $98^{27}$ program, and the AIMP calculations were performed using the MOLCAS4 $4^{28}$ program. The spectroscopic constants were determined by fitting the Morse potential for 10 (or 11) points near the equilibrium distance with a spacing of $0.1 a_{0}$.

\section{RESULTS AND DISCUSSION}

\section{A. Atomic calculations}

First, we present the atomic results of the $\mathrm{Pt}, \mathrm{Au}$, and $\mathrm{Hg}$ atoms in order to check the quality of the AIMP. Table I shows the expected radial values of the valence orbitals. The values calculated by the RESC-AIMP method are in excellent agreement with those found by the all-electron RESC method. Comparison with the nonrelativistic AIMP results shows a change in the orbital (radial) part of the wave function due to relativistic effects. It is the core that is expected to be greatly changed by these relativistic effects. One can expect the core to shrink and the valence $6 s$ and $5 p$ orbitals to may be shrink, and $5 d$ orbitals to expand to accommodate the change in the core density.

In Table II, we show the ionization energies, electron affinities, and interconfigurational energies. The discrepancies between the all-electron RESC and the RESC-AIMP results are less than $0.03 \mathrm{eV}$. At the $\mathrm{HF}$ level, in particular, the error is less than $0.01 \mathrm{eV}$ because the spectral representation operator reproduces almost the same exchange operator as that of the all-electron RESC method. The valence $6 \mathrm{~s}$ and $5 p$ orbitals are stabilized, while the $5 d$ orbital is destabilized, due to a relativistic effect. This leads to an increase 
TABLE II. Ionization potentials, electron affinities, and interconfigurational energies (eV).

\begin{tabular}{|c|c|c|c|c|c|c|}
\hline \multirow[b]{2}{*}{ Method } & \multicolumn{2}{|c|}{ Ionization potential } & \multicolumn{2}{|c|}{ Electron affinity } & \multicolumn{2}{|c|}{ Interconfigurational energy } \\
\hline & $\mathrm{HF}$ & CCSD & $\mathrm{HF}$ & CCSD & $\mathrm{HF}$ & CCSD \\
\hline $\mathrm{Pt}$ & \multicolumn{2}{|c|}{$s^{1} d^{9} \rightarrow s^{0} d^{9}$} & \multicolumn{2}{|c|}{$s^{1} d^{9} \rightarrow s^{2} d^{9}$} & \multicolumn{2}{|c|}{$s^{1} d^{9} \rightarrow s^{2} d^{8}$} \\
\hline RESC-AE & 7.55 & 8.71 & 0.20 & 1.68 & 0.80 & 0.50 \\
\hline RESC-AIMP & 7.55 & 8.73 & 0.20 & 1.68 & 0.81 & 0.50 \\
\hline NR-AIMP & 5.95 & 6.92 & -0.51 & 0.71 & 3.56 & 3.24 \\
\hline $\mathrm{Au}$ & \multicolumn{2}{|c|}{$s^{1} d^{10} \rightarrow s^{0} d^{10}$} & \multicolumn{2}{|c|}{$s^{1} d^{10} \rightarrow s^{2} d^{10}$} & \multicolumn{2}{|c|}{$s^{1} d^{10} \rightarrow s^{2} d^{9}$} \\
\hline RESC-AE & 7.55 & 8.83 & 0.42 & 1.85 & 1.96 & 1.69 \\
\hline RESC-AIMP & 7.55 & 8.86 & 0.42 & 1.86 & 1.97 & 1.66 \\
\hline NR-AIMP & 5.91 & 6.91 & -0.39 & 0.80 & 5.07 & 4.73 \\
\hline $\mathrm{Hg}$ & \multicolumn{2}{|c|}{$s^{2} d^{10} \rightarrow s^{1} d^{10}$} & $\cdots$ & $\cdots$ & $\cdots$ & $\cdots$ \\
\hline $\mathrm{RESC}-\mathrm{AE}$ & 8.40 & 9.99 & $\cdots$ & $\cdots$ & $\cdots$ & $\cdots$ \\
\hline RESC-AIMP & 8.40 & 10.01 & $\cdots$ & $\cdots$ & $\cdots$ & $\cdots$ \\
\hline NR-AIMP & 6.34 & 8.20 & $\cdots$ & $\ldots$ & $\cdots$ & $\ldots$ \\
\hline
\end{tabular}

in the ionization potentials and electron affinities and a decrease in the interconfigurational energies. The agreement between the RESC-AIMP and the all-electron RESC methods is excellent. Therefore, it can be concluded that the quality of the AIMP is sufficient at the atomic level.

\section{B. PtH, AuH, and $\mathrm{HgH}$}

To investigate the relationship between the valence basis set and the molecular properties, we performed HF and CCSD calculations on the hydride molecules of $\mathrm{Pt}, \mathrm{Au}$, and $\mathrm{Hg}$, and calculated the spectroscopic constants. The results for the bond lengths $R_{e}$, vibrational frequencies $\omega_{e}$, and dissociation energies $D_{e}$ are shown in Table III.

For $\mathrm{AuH}$, the reference all-electron results are: $R_{e}$ $=1.583 \AA ; \omega_{e}=2055 \mathrm{~cm}^{-1} ;$ and $D_{e}=1.67 \mathrm{eV}$ at the $\mathrm{HF}$ level, and $R_{e}=1.540 \AA$; $\omega_{e}=2225 \mathrm{~cm}^{-1}$; and $D_{e}=2.83 \mathrm{eV}$ at the CCSD level. The correlation effect decreases $R_{e}$ and increases $\omega_{e}$ and $D_{e}$. The differences between the AIMP and all-electron RESC calculations with the same basis set are estimated to be $0.004 \AA$ for $R_{e},-12 \mathrm{~cm}^{-1}$ for $\omega_{e}$, and $-0.02 \mathrm{eV}$ for $D_{e}$ at the HF level. The corresponding errors are almost negligible at the CCSD level. The AIMP with the reduced number of primitives $(13 s 10 p 9 d 6 f)$ of Ref. 7 gives slightly larger errors: $0.011 \AA$; $-23 \mathrm{~cm}^{-1}$; and $-0.06 \mathrm{eV}$ at the HF level, and $0.009 \AA ;-35 \mathrm{~cm}^{-1}$; and $-0.06 \mathrm{eV}$ at the CCSD level. The errors of the AIMP method with the valence-only contracted basis set are similar to those with the uncontracted basis sets at the HF level: $0.007 \AA$; $-25 \mathrm{~cm}^{-1}$; and $-0.06 \mathrm{eV}$. However, the errors become noticeable at the CCSD level: $0.012 \AA ;-64 \mathrm{~cm}^{-1}$; and $-0.10 \mathrm{eV}$. Introduction of the AIMP approximation tends to increase $R_{e}$ and decrease $\omega_{e}$ and $D_{e}$ at both the HF and the CCSD levels.

A comparison of $\mathrm{HF}$ and CCSD results with and without correction of the BSSE clearly shows the need to include this correction. The BSSE is rather small at the HF level, but it becomes significant at the CCSD level. Comparison of the BSSE corrected and uncorrected values shows that the BSSE correction increases $R_{e}$ and decreases $\omega_{e}$ and $D_{e}$ at both the $\mathrm{HF}$ and CCSD levels. This tendency is common to both the all-electron and AIMP calculations. We should note that the counterpoise method overestimates the BSSE as pointed out by several authors. ${ }^{29}$

Very similar trends to those discussed above can be observed in the calculations of $\mathrm{PtH}$ and $\mathrm{HgH}$.

The present results indicate that the AIMP method can reproduce the spectroscopic constants of the reference allelectron RESC calculations, provided that the valence basis sets are sufficiently large.

TABLE III. Spectroscopic constants of $\operatorname{AuH}\left({ }^{1} \Sigma^{+}\right), \operatorname{HgH}\left({ }^{2} \Sigma^{+}\right)$, and $\operatorname{PtH}\left({ }^{2} \Sigma^{+}\right)$. Results without counterpoise correction are given in parentheses.

\begin{tabular}{|c|c|c|c|c|c|c|c|c|c|c|}
\hline \multirow[b]{2}{*}{ Method } & \multirow[b]{2}{*}{ Basis set } & \multicolumn{3}{|c|}{$\mathrm{AuH}$} & \multicolumn{3}{|c|}{$\mathrm{PtH}$} & \multicolumn{3}{|c|}{$\mathrm{HgH}$} \\
\hline & & $R_{e}(\AA)$ & $\omega_{e}\left(\mathrm{~cm}^{-1}\right)$ & $D_{e}(\mathrm{eV})$ & $R_{e}(\AA)$ & $\omega_{e}\left(\mathrm{~cm}^{-1}\right)$ & $D_{e}(\mathrm{eV})$ & $R_{e}(\AA)$ & $\omega_{e}\left(\mathrm{~cm}^{-1}\right)$ & $D_{e}(\mathrm{eV})$ \\
\hline \multicolumn{11}{|l|}{$\mathrm{HF}$} \\
\hline $\mathrm{AE}$ & (22.17.14.9) & $1.583(1.580)$ & 2055 (2067) & 1.67 (1.69) & $1.550(1.549)$ & 2187 (2196) & $1.93(1.95)$ & $1.718(1.714)$ & $1650(1656)$ & $0.03(0.05)$ \\
\hline AIMP & (22.17.14.9) & $1.587(1.584)$ & 2043 (2049) & $1.65(1.66)$ & $1.554(1.552)$ & $2174(2180)$ & $1.91(1.92)$ & $1.719(1.716)$ & $1652(1651)$ & $0.03(0.04)$ \\
\hline AIMP & (13.10.9.6) & $1.594(1.591)$ & $2032(2036)$ & $1.61(1.62)$ & $1.554(1.551)$ & $2163(2173)$ & $1.87(1.89)$ & $1.715(1.711)$ & $1662(1663)$ & $0.04(0.04)$ \\
\hline AIMP & $\begin{array}{l}(22.17 .14 .9) / \\
{[4.4 .4 .2]}\end{array}$ & $1.590(1.588)$ & $2030(2037)$ & $1.61(1.63)$ & $1.556(1.555)$ & 2157 (2163) & $1.87(1.88)$ & $1.727(1.724)$ & $1608(1608)$ & $-0.02(-0.01)$ \\
\hline \multicolumn{11}{|l|}{ CCSD } \\
\hline $\mathrm{AE}$ & (22.17.14.9) & $1.540(1.530)$ & 2225 (2259) & $2.83(2.94)$ & $1.524(1.515)$ & 2379 (2408) & $3.21(3.34)$ & $1.763(1.737)$ & 1263 (1348) & $0.28(0.39)$ \\
\hline AIMP & (22.17.14.9) & $1.540(1.529)$ & $2222(2252)$ & $2.84(2.94)$ & $1.525(1.515)$ & 2371 (2398) & $3.20(3.32)$ & $1.762(1.735)$ & 1264 (1344) & $0.29(0.38)$ \\
\hline AIMP & (13.10.9.6) & $1.549(1.536)$ & $2190(2230)$ & $2.77(2.89)$ & $1.527(1.513)$ & $2350(2395)$ & $3.16(3.31)$ & $1.757(1.723)$ & 1277 (1383) & $0.29(0.41)$ \\
\hline AIMP & $\begin{array}{l}(22.17 .14 .9) / \\
{[4.4 .4 .2]}\end{array}$ & $1.552(1.539)$ & $2161(2198)$ & $2.73(2.89)$ & $1.533(1.520)$ & $2327(2360)$ & $3.14(3.31)$ & $1.787(1.749)$ & $1193(1312)$ & $0.26(0.40)$ \\
\hline
\end{tabular}


TABLE IV. Spectroscopic constants of $\mathrm{Au}_{2}$. Results without counterpoise correction are given in parentheses.

\begin{tabular}{lcclccc}
\hline \hline \multicolumn{1}{c}{ Method } & Active & Basis set & Reference & $R_{e}(\AA)$ & $\omega_{e}\left(\mathrm{~cm}^{-1}\right)$ & $D_{e}(\mathrm{eV})$ \\
\hline NR-AIMP CCSD $(\mathrm{T})$ & $22 e^{-}$ & $(21.17 .11 .7) /[11.9 .8 .5]$ & This work & $2.774(2.766)$ & $121(122)$ \\
RESC-AIMP CCSD(T) & $22 e^{-}$ & $(21.17 .11 .7) /[11.9 .8 .5]$ & This work & $2.524(2.516)$ & $178(180)$ \\
RESC-AIMP CCSD(T) & $34 e^{-}$ & $(21.17 .11 .7) /[11.9 .8 .5]$ & This work & $2.511(2.499)$ & $183(185)$ & $2.03(2.08)$ \\
DK-AE CCSD(T) $)^{\mathrm{a}}$ & $22 e^{-}$ & $(21.17 .11 .7) /[12.11 .6 .4]$ & 30 & 2.521 & $2.12(2.19)$ \\
PP CCSD(T) & $38 e^{-}$ & $(11.9 .8 .4) /[10.8 .7 .4]$ & 31 & 2.505 & 180 & 182 \\
Expt. & $\cdots$ & $\ldots$ & 32 & 2.472 & 191 & 2.04 \\
\hline
\end{tabular}

${ }^{\mathrm{a} B S S E-c o r r e c t e d}$.

${ }^{\mathrm{b}}$ BSSE-uncorrected.

\section{C. $\mathrm{Au}_{2}$}

$\mathrm{Au}_{2}$ is probably the most important benchmark molecule for testing the relativistic methods. Although extensive studies have been performed using this molecule, it is still difficult to predict the spectroscopic properties of $\mathrm{Au}_{2}$ reliably. Table IV shows the CCSD(T) results for $\mathrm{Au}_{2}$. Some previous theoretical values, ${ }^{30,31}$ as well as experimental values, ${ }^{32}$ are also listed in Table IV for comparison. The effects of the BSSE on spectroscopic constants were also investigated. Nonrelativistic AIMP calculations were also carried out, and results are shown in Table IV. Relativistic effects on the spectroscopic constants of $\mathrm{Au}_{2}$ are significant, which is now well understood, and the trend is consistent with previous calculations. ${ }^{33-35}$

We shall begin with a discussion of the BSSE. This important aspect has sometimes been overlooked. In the case of the ANO-type basis set $[11 s 9 p 8 d 5 f]$, the BSSE are -0.008 $\AA,+2 \mathrm{~cm}^{-1}$, and $+0.05 \mathrm{eV}$ for $R_{e}, \omega_{e}$, and $D_{e}$, respectively. Since no completely reliable scheme for eliminating the BSSE exists, the choice of the valence-only basis set is very important for the accurate determination of molecular properties.

The RESC-AIMP method gives the same quality of results $\left(2.524 \AA, 178 \mathrm{~cm}^{-1}\right.$, and $2.03 \mathrm{eV}$ for $R_{e}, \omega_{e}$, and $D_{e}$, respectively) as the all-electron Douglas-Kroll method $\left(2.521 \AA, 180 \mathrm{~cm}^{-1}, 2.04 \mathrm{eV}\right)$, when the basis set and the electronic correlation are treated at the same level. The best results we obtained were at the $\operatorname{CCSD}(\mathrm{T})$ level, with the 34 valence electrons correlated: $R_{e}=2.511 \AA, \omega_{e}=183 \mathrm{~cm}^{-1}$, and $D_{e}=2.12 \mathrm{eV}$. These values are in satisfactory agreement with experimental data: $R_{e}=2.472 \AA, \omega_{e}=191 \mathrm{~cm}^{-1}$, and $D_{e}=2.29 \mathrm{eV}$. Further improvement may require an extensive basis set with angular momentum functions higher than the $f$ level, as pointed out by Hess and Kaldor. ${ }^{30}$

\section{D. $\mathrm{Hg}_{2}$}

$\mathrm{Hg}_{2}$ is known as a weakly bound van der Waals molecule, so highly correlated methods with the extensive basis set and a BSSE correction, as well as relativistic effects, are required to reliably determine the spectroscopic constants. Table V shows the spectroscopic constants of $\mathrm{Hg}_{2}$ calculated at the $\operatorname{CCSD}(\mathrm{T})$ level. In the present $\operatorname{CCSD}(\mathrm{T})$ calculations, the $5 p, 5 d$, and $6 s$ electrons were correlated. Selected results of previous theoretical studies ${ }^{36,37}$ are also summarized in Table V.

The inclusion of relativistic effects at the $\operatorname{CCSD}(\mathrm{T})$ level decreases $R_{e}$ by $0.259 \AA$ and increases $\omega_{e}$ by $0.8 \mathrm{~cm}^{-1}$ and $D_{e}$ by $0.001 \mathrm{eV}$. These tendencies are in agreement with the previous calculations by $\mathrm{Yu}$ et al. ${ }^{37}$

The best result obtained was $3.836 \AA, 16.3 \mathrm{~cm}^{-1}$, and $0.039 \mathrm{eV}$ for $R_{e}, \omega_{e}$, and $D_{e}$, respectively. The discrepancies with the experimental values ${ }^{38,39}$ are about $+0.2 \AA,-2$ $\mathrm{cm}^{-1}$, and $-0.004 \mathrm{eV}$. Our results are satisfactory at this level of theory. We did not include any spin-orbit effects. Dolg and $\mathrm{Flad}^{40}$ reported that the spin-orbit effects decrease $R_{e}$ by $0.035 \AA$ and increase $D_{e}$ by $0.003 \mathrm{eV}$. However, these effects are of the same order of magnitude as the experimental error bars.

\section{E. $\mathrm{Pt}_{2}$}

In spite of various theoretical ${ }^{41-43}$ and experimental ${ }^{44-48}$ studies, the molecular properties of $\mathrm{Pt}_{2}$ are not well known. In particular, the identity of the ground state electronic structure of this molecule is still unresolved, which leads to difficulty in interpreting experimental observations. In this study, the lowest 12 electronic states were calculated in both the singlet and triplet manifold in each representation in $D_{2 h}$. All calculations were performed with the AIMP

TABLE V. Spectroscopic constants of $\mathrm{Hg}_{2}$. Results without counterpoise correction are given in parentheses.

\begin{tabular}{|c|c|c|c|c|c|c|}
\hline Method & Active & Basis set & Reference & $R_{e}(\AA)$ & $\omega_{e}\left(\mathrm{~cm}^{-1}\right)$ & $D_{e}(\mathrm{eV})$ \\
\hline NR-AIMP CCSD(T) & $36 e^{-}$ & $(24.17 .14 .8) /[10.8 .7 .4]$ & This work & $4.244(4.205)$ & $14.7(14.8)$ & $0.035(0.037)$ \\
\hline RESC-AIMP CCSD(T) & $36 e^{-}$ & $(24.17 .14 .8) /[10.8 .7 .4]$ & This work & $3.985(3.920)$ & $15.5(16.1)$ & $0.034(0.037)$ \\
\hline RESC-AIMP CCSD(T) & $36 e^{-}$ & $(24.17 .14 .8) /[15.10 .8 .5]$ & This work & $3.836(3.720)$ & $16.3(17.8)$ & $0.039(0.048)$ \\
\hline PP QCISD(T)/MP2 & $40 e^{-}$ & $(9.8 .7 .2)$ & 36 & $4.15(3.93)$ & (14) & $0.022(0.043)$ \\
\hline $\operatorname{PP} \operatorname{CCSD}(T)^{\mathrm{a}}$ & $40 e^{-}$ & (9.8.7.5.5) & 37 & 3.769 & 19 & 0.044 \\
\hline \multirow[t]{2}{*}{ Expt. } & $\cdots$ & $\ldots$ & 38 & $3.63 \pm 0.04$ & $18.5 \pm 0.5$ & - \\
\hline & $\cdots$ & $\cdots$ & 39 & - & $19 \pm 2$ & $0.043 \pm 0.003$ \\
\hline
\end{tabular}

${ }^{a}$ BSSE-corrected and including spin-orbit effects. 
TABLE VI. Spectroscopic constants of the $\mathrm{Pt}_{2}$ ground state.

\begin{tabular}{lllcc}
\hline \hline \multicolumn{1}{c}{ Method } & Reference & $R_{e}(\AA)$ & $\omega_{e}\left(\mathrm{~cm}^{-1}\right)$ & $D_{e}(\mathrm{eV})$ \\
\hline 4-Comp. RGGA $^{\mathrm{a}}$ & 41 & 2.39 & 239 & 3.94 \\
PP CASPT2 $^{\text {PP CASSCF-FOCI }}$ & 42 & 2.409 & - & 2.62 \\
PP CASSCF-FOCI+SO $^{\mathrm{b}}$ & 43 & 2.428 & 219 & 2.27 \\
RESC-AIMP CASPT2 $^{\mathrm{c}}$ & 43 & 2.456 & 189 & 1.97 \\
Expt. $^{\text {This work }}$ & 2.293 & 256 & 3.82 \\
& 44 & 2.34 & 259 & $3.71 \pm 0.16$ \\
& 45 & - & - & - \\
& 46 & $-45 \pm 0.26$ & - & - \\
& 47 & - & $215 \pm 15$ & - \\
\hline \hline
\end{tabular}

${ }^{\mathrm{a}}$ Four-components relativistic generalized gradient approximation.

${ }^{\mathrm{b}}$ Including spin-orbit (SO) effects.

${ }^{c}$ BSSE-corrected.

[9s7p6d4f] basis set at the CASPT2 level. The 32 active electrons were distributed among the 18 active orbitals for the reference CASSCF wave functions. The dissociation energies were calculated with respect to the atomic $s^{1} d^{9}\left({ }^{3} D\right)$ state. The ground state was calculated to be the ${ }^{3} B_{1 g}\left({ }^{3} \Sigma_{g}^{-}\right)$ state arising from the $\left(\pi_{g}\right)^{2}$ configuration with a bond length of $2.289 \AA$ and a vibrational frequency of $259 \mathrm{~cm}^{-1}$, and the binding energy was estimated to be $3.89 \mathrm{eV}$. The other 11 electronic states, arising from different configurations with the partially occupied $5 d$ orbitals, exist within a very narrow energy range $(1.16 \mathrm{eV})$. The first excited state was computed to be the ${ }^{3} B_{3 u}\left({ }^{3} \Pi_{u}\right)$ state $\left(R_{e}=2.308 \AA, \omega_{e}=248 \mathrm{~cm}^{-1}\right.$, $\left.D_{e}=3.69 \mathrm{eV}\right)$, arising from the $\left(\sigma_{u}\right)^{1}\left(\pi_{g}\right)^{3}$ configuration, lying $0.20 \mathrm{eV}$ above the ground state, and the ${ }^{1} B_{3 u}\left({ }^{1} \Pi_{u}\right)$ state $\left(R_{e}=2.313 \AA, \omega_{e}=240 \mathrm{~cm}^{-1}, D_{e}=3.44 \mathrm{eV}\right)$ was the lowest singlet state, $0.45 \mathrm{eV}$ above the ground state.

For the lowest ${ }^{3} B_{1 g}\left({ }^{3} \Sigma_{g}^{-}\right)$state, we performed CASPT2 calculations with the extended basis set of [13s $10 p 8 d 5 f]$. The calculated results are summarized in Table VI with experimental and previous theoretical values. The calculated spectroscopic constants of the ground state were found to be $2.293 \AA, 256 \mathrm{~cm}^{-1}$, and $3.82 \mathrm{eV}$ for $R_{e}, \omega_{e}$, and $D_{e}$, respectively. There are several experimental data. Our computed results support the ones observed by Gupta et al. ${ }^{44}$ The assignment of the ground states agrees with the previous calculations of Cui et al. ${ }^{42}$ They reported that the spectroscopic constants of the ground state ${ }^{3} B_{1 g}\left({ }^{3} \Sigma_{g}^{-}\right)$were $2.409 \AA$ and $2.62 \mathrm{eV}$ for $R_{e}$ and $D_{e}$, respectively, at the CASPT2 level. Balasubramanian ${ }^{43}$ also reported that by considering spinorbit effects at the first-order configuration interaction (FOCI) level, the ${ }^{3} \Sigma_{g}^{-}$state is the lowest energy state and the ${ }^{3} \Gamma_{u}$ state lies $0.13 \mathrm{eV}$ above this. With respect to the ${ }^{3} \Sigma_{g}^{-}$ state, spin-orbit effects increase $R_{e}$ by $0.028 \AA$, decrease $\omega_{e}$ by $30 \mathrm{~cm}^{-1}$, and decrease $D_{e}$ by $0.30 \mathrm{eV}$. Taking account of the spin-orbit effects, our results at the spin-free level seem very encouraging.

\section{CONCLUSIONS}

A RESC-AIMP method has been developed by modifying the one-electron operator in the usual AIMP method, and the AIMPs for $\mathrm{Pt}, \mathrm{Au}$, and $\mathrm{Hg}$ atoms were generated. We have shown that the RESC-AIMP method can reproduce the spectroscopic constants of the reference all-electron RESC calculations, provided that the valence basis sets are sufficiently large.

We have applied the method successfully to the study of metal dimers using the $\operatorname{CCSD}(\mathrm{T})$ and CASPT2 methods. For $\mathrm{Au}_{2}$, the present scheme shows the same qualities as the previous all-electron Douglas-Kroll calculation. For $\mathrm{Hg}_{2}$, as indicated by other authors, a large basis set and the correction of the BSSE, as well as the correlation, are indispensable in calculating accurate spectroscopic constants. We have concluded that the ground state of $\mathrm{Pt}_{2}$ is the ${ }^{3} \Sigma_{g}^{-}$state, and have presented reliable spectroscopic constants at the spinfree level of theory.

It is concluded that RESC-AIMP is an accurate and efficient alternative to more demanding all-electron relativistic methods.

\section{ACKNOWLEDGMENTS}

The present research is supported in part by a grant-inaid for Scientific Research on Priority Areas "Molecular Physical Chemistry" from the Ministry of Education, Science, Culture, and Sports of Japan, and by a grant from the Genesis Research Institute. Partial support from MEC, Spain (Grant No. DGESIC, PB98-0108) is also acknowledged.

${ }^{1}$ J. C. Philips and L. Kleinman, Phys. Rev. 116, 287 (1959).

${ }^{2}$ V. Bonifacic and S. Huzinaga, J. Chem. Phys. 60, 2779 (1974).

${ }^{3}$ S. Huzinaga, L. Seijo, Z. Barandiarán, and M. Klobukowski, J. Chem. Phys. 86, 2132 (1987).

${ }^{4}$ L. Seijo, Z. Barandiarán, and S. Huzinaga, J. Chem. Phys. 91, 7011 (1989).

${ }^{5}$ Z. Barandiarán and L. Seijo, Can. J. Chem. 70, 409 (1992).

${ }^{6}$ Z. Barandiarán, L. Seijo, and S. Huzinaga, J. Chem. Phys. 93, 5843 (1990)

${ }^{7}$ M. Casarrubios and L. Seijo, J. Chem. Phys. 110, 784 (1999).

${ }^{8}$ L. Seijo, J. Chem. Phys. 102, 8078 (1995).

${ }^{9}$ M. Casarrubios and L. Seijo, J. Mol. Struct.: THEOCHEM 426, 59 (1998).

${ }^{10}$ C. Wittborn and U. Wahlgren, Chem. Phys. 201, 357 (1995).

${ }^{11}$ F. Rakowitz, C. M. Marian, L. Seijo, and U. Wahlgren, J. Chem. Phys. 110, 3678 (1999).

${ }^{12}$ F. Rakowitz, C. M. Marian, and L. Seijo, J. Chem. Phys. 111, 10436 (1999)

${ }^{13}$ T. Nakajima and K. Hirao, Chem. Phys. Lett. 302, 383 (1999). 
${ }^{14}$ T. Nakajima, T. Suzumura, and K. Hirao, Chem. Phys. Lett. 304, 271 (1999).

${ }^{15}$ T. Suzumura, T. Nakajima, and K. Hirao, Int. J. Quantum Chem. 75, 757 (1999).

${ }^{16}$ T. Nakajima, K. Koga, and K. Hirao, J. Chem. Phys. 112, 10142 (2000).

${ }^{17}$ H. A. Witek, T. Nakajima, and K. Hirao, J. Chem. Phys. 113, 8015 (2000).

${ }^{18}$ R. J. Buenker, P. Chandra, and B. A. Hess, Chem. Phys. 84, 1 (1984).

${ }^{19}$ K. Fægri and J. Almlöf, J. Comput. Chem. 7, 396 (1986).

${ }^{20}$ D. P. Chong and S. R. Langhoff, J. Chem. Phys. 84, 5606 (1986)

${ }^{21}$ D. G. C. Lie and E. Clementi, J. Chem. Phys. 60, 1275 (1974).

${ }^{22}$ S. F. Boys and F. Bernardi, Mol. Phys. 19, 553 (1970).

${ }^{23}$ S. A. Pizlo, G. Jansen, B. A. Heß, and W. von Niessen, J. Chem. Phys. 98, 3945 (1993).

${ }^{24}$ P.-O. Widmark, P. A. Malmqvist, and B. O. Roos, Theor. Chim. Acta 77, 291 (1990)

${ }^{25}$ P.-O. Widmark, B. J. Persson, and B. O. Roos, Theor. Chim. Acta 79, 419 (1991).

${ }^{26}$ R. Pou-Amérigo, M. Merchan, I. Nebot-Grl, P.-O. Widmark, and B. O. Roos, Theor. Chim. Acta 92, 149 (1995).

${ }^{27}$ MOLPRO is a package of ab initio programs written by H. J. Werner and P. J. Knowles, with contributions from J. Almlof et al.

${ }^{28} \mathrm{~K}$. Andersson et al., MOLCAS version 4, University of Lund, Sweden (1994).

${ }^{29}$ K. Morokuma and K. Kitaura, in Chemical Application of Atomic and Molecular Electronic Potentials, edited by P. Politzer (Plenum, New York, 1981).

${ }^{30}$ B. A. Hess and U. Kaldor, J. Chem. Phys. 112, 1809 (2000).

${ }^{31}$ R. Wesendrup, J. K. Laerdahl, and P. Schwerdtfeger, J. Chem. Phys. 110, 9457 (1999)
${ }^{32}$ K. P. Huber and G. Herzberg, Molecular Spectra and Molecular Structure Constants of Diatomic Molecules (Van Nostrand, New York, 1979).

${ }^{33}$ D. Strömberg and U. Wahlgren, Chem. Phys. Lett. 169, 109 (1990).

${ }^{34}$ P. Schwerdtfeger, Chem. Phys. Lett. 183, 457 (1991).

${ }^{35}$ R. Wesendrup, J. K. Laerdahl, and P. Schwerdtfeger, J. Chem. Phys. 110, 9457 (1999)

${ }^{36}$ P. Schwerdtfeger, J. Li, and P. Pyykkö, Theor. Chim. Acta 87, 313 (1994).

${ }^{37}$ M. Yu and M. Dolg, Chem. Phys. Lett. 273, 329 (1997).

${ }^{38}$ R. D. van Zee, S. C. Blankespoor, and T. S. Zwier, J. Chem. Phys. 88, 4650 (1988).

${ }^{39}$ A. Zehnacker, M. C. Duval, C. Jouvet, C. Lardeux-Dedonder, D. Solgadi, B. Soep, and O. B. d'Azy, J. Chem. Phys. 86, 6565 (1987).

${ }^{40}$ M. Dolg and H.-J. Flad, J. Phys. Chem. 100, 6147 (1996).

${ }^{41}$ V. Varge, B. Fricke, H. Nakamatsu, J. Anton, D. Geschke, A. Heitmann, E. Engel, and T. Bastug, J. Chem. Phys. 112, 3499 (2000).

${ }^{42}$ Q. Cui, D. G. Musaev, and K. Morokuma, J. Chem. Phys. 108, 8418 (1998).

${ }^{43}$ K. Balasubramanian, J. Chem. Phys. 87, 6573 (1987).

${ }^{44}$ S. K. Gupta, B. M. Nappi, and K. A. Gingerich, Inorg. Chem. 20, 966 (1981).

${ }^{45}$ S. Taylor, G. W. Lemire, Y. M. Hamrick, Z. Fu, and M. D. Morse, J. Chem. Phys. 89, 5517 (1988)

${ }^{46}$ J. Xhie, K. Sattler, U. Muller, N. Venkateswaran, and G. Raina, J. Vac. Sci. Technol. B 9, 829 (1991).

${ }^{47}$ J. Ho, M. L. Polak, K. M. Ervin, and W. C. Lineberger, J. Chem. Phys. 99, 8542 (1993).

${ }^{48}$ H. Wang, Y. Liu, H. Haouari, R. Craig, J. R. Lombardi, and D. M. Lindsay, J. Phys. Chem. 101, 7036 (1997). 